\title{
Definições operacionais das características definidoras do Diagnóstico de Enfermagem Volume de Líquidos Excessivo*
}

\author{
Operational definitions of the defining characterístics of the nursing \\ diagnosis Fluid Volume Excess \\ Definiciones operacionales de las características definidoras del
diagnóstico de enfermería Volumen de Líquídos Excesivo
}

Rita Narriman Silva de Oliveira Boery ${ }^{1}$, Heloisa Cristina Quatrini Carvalho Passos Guimarães², Alba Lúcia Bottura Leite de Barros ${ }^{3}$

\begin{abstract}
RESUMO: As definições operacionais são requisitos importantes no ensino da Semiologia e Semiotécnica nos Cursos de Graduação e Pós-Graduação em Enfermagem. Este estudo descritivo, acerca das definições operacionais elaboradas para as características definidoras do diagnóstico de enfermagem Volume de Líquidos Excessivo em portadores de cardiopatias foi realizado a partir de revisão da literatura, no período de 1985 a 2001. As definições operacionais foram analisadas criticamente por quatro enfermeiros especialistas em diferentes campos clínicos de atuação. Após os ajustes sugeridos por esses especialistas, os dados foram enviados a uma consultora técnica, para avaliação conclusiva.
\end{abstract}

Descritores: Diagnóstico de enfermagem; Edema

ABSTRACT: Operational definitions are important tools for teaching Semiology and Semiotechuiques both for undergraduate and graduate nursing students. This descriptive study on the operational definitions of the defining characteristics of nursing diagnosis Fluid Volume Excess in cardiac patients was based on literature review regarding the period from 1985 to 2001. The operational definitions were analyzed by four expert nurses from different clinical areas. After the suggested corrections were made, the data was submitted to a technical consultant for final evaluation.

Descriptors: Nursing diagnosis; Edema

RESUMEN: Las definiciones operacionales son subsidios importantes para la ensveñanza de Semiologia y Semiotécnica en los cursos de pre y pós grado. Este estudio descriptivo, aurea de las caracteristicas definidoras del diagnóstico de enfermaria Volumen de Liquidos Excesivo en pacientes cardíacos, que realizado a partir de revision de literatura, del período de 1985 a 2001. las definiciones operacionales elaboradas fueron analizadas por cuatro enfermeros especialistas que actuavan en diferentes campos clínicos. Despues de realizar las correcciones sugeridas por esas especialistas, los dados fueron enviados a uma consultora técnica para evoluación final.

Descriptores: Diagnostico de enfermaría; Edema

\section{INTRODUÇÃo}

O ensino de Semiologia e Semiotécnica nos Cursos de Graduação e Pós-Graduação em Enfermagem, freqüentemente, utiliza as classificações do diagnóstico de enfer- magem como referência norteadora para o seu desenvolvimento. Este estudo faz uma proposta de definições operacionais das características definidoras do diagnóstico de enfermagem Volume de Líquidos Excessivo, esperando que elas possam contribuir para o ensino e a

\footnotetext{
* Extraído da Tese de Doutorado "Avaliação Clínica realizada pela enfermeira para estabelecer o diagnóstico de Enfermagem Volume de Líquidos Excessivo em portadores de cardiopatias." apresentada à UNIFESP, vinculada ao Projeto de Integração Multidisciplinar no Ensino da Semiologia e Semiotécnica como base para a assistência de enfermagem- PROIN (CAPES/ Departamento de Enfermagem)

1 Enfermeira. Professora Doutora do Departamento de Saúde da Universidade Estadual do Sudoeste da Bahia. E-mail: rboery@ig.com.br

2 Enfermeira. Pesquisadora Científica III do Instituto Lauro de Souza Lima. Doutora em Enfermagem-UNIFESP. E-mail: clinicapes@ilsl.br

3 Enfermeira. Doutora em Ciências pela Escola Paulista de Medicina. Professor Adjunto do Departamento de Enfermagem da UNIFESP. Diretora de Enfermagem do Hospital São Paulo-UNIFESP.
} 
prática profissional da enfermeira e, ainda, que possam servir como modelo e ser aprofundadas em outros estudos, subsidiando assim a pesquisa nesta área.

As definições operacionais dão significado prático às definições conceituais, especificando as atividades e procedimentos necessários para se avaliar essas características definidoras.

O diagnóstico de enfermagem Volume de Líquidos Excessivo é definido como o estado no qual um indivíduo experimenta uma retenção aumentada de líquidos isotônicos ${ }^{(1)}$. Os fatores relacionados a este diagnóstico são: comprometimento dos mecanismos reguladores, excessiva ingestão de líquidos e excessiva ingestão de sódio $^{(1)}$.

As características definidoras desse diagnóstico são: 1. ganho de peso num curto período 2 . ingesta ${ }^{(a)}$ maior do que o débito; 3. alterações da pressão arterial; 4. alterações da pressão da artéria pulmonar; 5. pressão venosa central aumentada; 6. edema; 7. anasarca; 8 . distensão da veia jugular; 9. mudança no padrão respiratório; 10. dispnéia ou respiração curta; 11 . ortopnéia; 12 . sons respiratórios anormais - ruídos adventícios, estertores e crepitações; 13 . congestão pulmonar; 14 . derrame pleural; 15. hemoglobina e hematócrito diminuídos; 16. eletrólitos alterados; 17 . alterações na densidade urinária; 18. som cardíaco B3; 19. reflexo hepatojugular positivo; 20. oligúria; 21 . azotemia; 22 . mudança no estado mental; 23. agitação; 24 . ansiedade $^{(1)}$.

Estas características são dados objetivos e/ou subjetivos que podem ser obtidos por procedimentos propedêuticos, aferição ou por apoio diagnóstico, seja laboratorial ou radiológico.

As definições operacionais são componentes essenciais das pesquisas de diagnóstico de enfermagem, porque elas preenchem uma lacuna entre a observação e a investigação científica. Elas descrevem o que será mensurado e como pode ser feita essa mensuração. Elas servem para: aumentar a confiabilidade e validade dos dados relacionada aos diagnósticos de enfermagem; facilitar a replicação da pesquisa; aumentar a habilidade do pesquisador em relacionar os achados a estudos anteriores; e para indicar os critérios para a avaliação das intervenções de enfermagem, ao se proceder à evolução do paciente $^{(2)}$.

O conhecimento obtido com a definição operacional deverá, portanto, servir para aumentar o entendimento do conceito teórico proposto, porque deve aproximar a teoria da prática $^{(3)}$.

\section{OBJETIVO}

- Elaborar definições operacionais das características definidoras do diagnóstico de enfermagem Volume de Líquidos Excessivo.

\section{MÉTODO}

A elaboração das definições operacionais foram embasadas nas definições conceituais de estudiosos ${ }^{(4-17)}$. Em seguida, a partir da nossa própria experiência, elaboramos definições operacionais das características definidoras do diagnóstico de enfermagem Volume de Líquidos Excessivo. Posteriormente, consultamos quatro enfermeiras experts, docentes das áreas de Semiologia e Semiotécnica, relacionadas ao Sistema Respiratório, Cardiovascular, Renal e à Saúde Mental, que deram o seu consentimento por escrito e foi solicitado que suas considerações fossem críticas no sentido de tornar as definições operacionais o mais próximo possível da prática profissional.

As experts foram selecionadas aleatoriamente, entre enfermeiras das diversas áreas pertinentes às características definidoras do diagnóstico de enfermagem Volume de líquidos Excessivo. Enviamos então, uma carta para cada uma delas, solicitando a colaboração. Para isso, anexamos uma cópia da proposta de definições feita preliminarmente. Depois de recolher essas contribuições e realizar os ajustes necessários, foi encaminhado para uma consultora técnica, uma estudiosa dessa área, para uma avaliação conclusiva. Somente depois disso, consideramos as definições operacionais elaboradas, mesmo assim, em forma de proposta, ou seja, sujeitas a novas alterações.

O estudo foi aprovado pelos Comitês de Ética e Pesquisa do Instituto Dante Pazzanese de Cardio-logia e do Hospital São Paulo/ Universidade Federal de São Paulo.

\section{RESULTADOS}

Para melhor visualizar os resultados das definições operacionais das características definidoras do diagnóstico de enfermagem Volume de Líquidos Excessivo estão exibidas no Quadro demonstrado a seguir.

\footnotetext{
(a) ingesta - embora este termo não conste em dicionários da Língua Portuguesa, ele será utilizado como foi denominado, na tradução do livro da NANDA (2002), por tratar-se da terminologia técnica consagrada em nosso meio.
} 
Quadro 1 - Proposta de definições operacionais das características definidoras do diagnóstico de enfermagem Volume de Líquidos Excessivo.

\begin{tabular}{|c|c|}
\hline $\begin{array}{l}\text { CARACTERÍSTICAS } \\
\text { DEFINIDORAS }\end{array}$ & DEFINIÇÃO OPERACIONAL \\
\hline Ganho de peso num curto período & $\begin{array}{l}\text { A mensuração diária do peso, com a mesma roupa, antes do desjejum e com a bexiga } \\
\text { esvaziada, deve ser feita com o auxilio de uma balança (instrumento) aferida em escala de } \\
\text { Quilograma, de preferência antropométrica, para permitir a mensuração da altura, medida pela } \\
\text { escala métrica. A informação sobre a duração de tempo em que o paciente mantém esse peso } \\
\text { deve ser questionada ao próprio paciente. Ele pode referir-se ao ajuste de roupas e sapatos, } \\
\text { sensação de mãos "grossas" e rígidas, percebida pela diminuição do tato, aperto de aliança e } \\
\text { anéis, sensação de "corpo mais pesado", entre outras informações. Um aumento de peso } \\
\text { superior a } 1 \mathrm{Kg} / \text { dia sugere a retenção de líquidos. }\end{array}$ \\
\hline Ingesta maior do que o débito & $\begin{array}{l}\text { A avaliação clínica de ingesta maior do que o débito faz-se pela aferição dos líquidos admi- } \\
\text { nistrados/ingeridos e eliminados devidamente registrados, utilizando-se instrumentos como } \\
\text { cálices/copos graduados, além da observação. A entrada de maior quantidade de líquidos no } \\
\text { organismo do que a saída (eliminação) caracteriza uma ingesta maior do que o débito. }\end{array}$ \\
\hline Alterações da pressão arterial & $\begin{array}{l}\text { A mensuração da pressão arterial, preferencialmente nos membros superiores, deve ser aferida } \\
\text { para avaliar alterações. Utiliza-se um esfigmomanômetro e o estetoscópio bi-auricular } \\
\text { (instrumentos) para tal procedimento. A informação do paciente, ou a leitura de registros } \\
\text { anteriores, são válidas para facilitar essa aferição. Se houver desvio para mais desses valores } \\
\text { habituais, a alteração da pressão arterial pode ser considerada. }\end{array}$ \\
\hline $\begin{array}{l}\text { Alterações da pressão da artéria } \\
\text { pulmonar }\end{array}$ & $\begin{array}{l}\text { A aferição da pressão da artéria pulmonar deve ser feita comparando-se o achado com o } \\
\text { registro de valores encontrados anteriormente, para considerar se há desvios. Se isso ocorrer, } \\
\text { pode ser caracterizada a alteração da pressão da artéria pulmonar. O valor considerado normal } \\
\text { para a pressão sistólica é de } 25 \mathrm{mmHg} \text {, e para a diastólica é de } 9 \mathrm{mmHg} \text {; o valor da média é } \\
\text { de } 15 \mathrm{mmHg} \text {. }\end{array}$ \\
\hline Pressão venosa central aumentada & $\begin{array}{l}\text { Para proceder-se à leitura da pressão venosa central o paciente deve estar em decúbito dorsal } \\
\text { horizontal e o nivelador precisa encontrar o ponto zero na escala métrica afixada, tomando por } \\
\text { base a linha médio-axilar ou o manúbrio external do paciente. Segue-se então a abertura da via } \\
\text { da torneira que permite a entrada da infusão venosa para o paciente, seguida da abertura da } \\
\text { via afixada à escala métrica e, ainda, da via da torneira que liga a afixada ao paciente, a fim } \\
\text { de obter a leitura que corresponde ao ponto de estabilidade da oscilação líquida, na escala } \\
\text { métrica afixada. Após a leitura, a torneira volta à primeira posição, de tal modo que o líquido } \\
\text { venoso flua para o paciente. A aferição da PVC mostrando uma tendência ascendente, } \\
\text { associada com outros achados clínicos do paciente, como estertores de base pulmonar, som } \\
\text { cardíaco S3, pulso e freqüência respiratória aumentando insidiosamente, pode ser um dado } \\
\text { mais significativo do que o valor encontrado isoladamente, e constitui-se como o meio de avaliar } \\
\text { se a PVC está aumentada. }\end{array}$ \\
\hline Edema & $\begin{array}{l}\text { Comprova-se o edema subcutâneo pela depressão, à qual dá-se o nome de fóvea ou cacifo. } \\
\text { A pele da região edemaciada torna-se lisa e brilhante quando o edema é recente. Com o } \\
\text { passar do tempo, a pele adquire o aspecto de "casca de laranja", indicando espessamento } \\
\text { com as retrações puntiformes correspondentes aos folículos pilosos. Ao exame físico, } \\
\text { portanto, utiliza-se a inspeção e a palpação. }\end{array}$ \\
\hline Anasarca & $\begin{array}{l}\text { A investigação de edema subcutâneo pela depressão deve ser feita nas extremidades, } \\
\text { acompanhada da inspeção da face, à procura de edema periorbital.; Deve-se realizar também } \\
\text { a ausculta pulmonar, em busca de estertores pulmonares e a pesquisa de ascite. Para avaliar } \\
\text { uma possível ascite deve-se fazer a percussão das regiões inferiores do abdome. Como o } \\
\text { líquido ascítico cai de modo característico devido à gravidade, enquanto as alças do intestino } \\
\text { repletas de gases flutuam na parte superior, é esperado um som maciço. Também se pode } \\
\text { utilizar a palpação pelo "Teste da onda líquida", que consiste em percutir um flanco e, com a } \\
\text { mão espalmada no flanco oposto, tentar sentir o impulso transmitido pelo líquido, em forma de } \\
\text { onda. A medida da circunferência abdominal também é útil na avaliação da evolução de ascite. }\end{array}$ \\
\hline Distensão da veia jugular & $\begin{array}{l}\text { A inspeção do paciente, que deve estar em decúbito dorsal a } 45^{\circ} \text {, é a forma de avaliar-se } \\
\text { a distensão da veia jugular. Para se formar um ângulo de } 45^{\circ} \text { entre as costas do paciente e o } \\
\text { leito, pode-se levantar a cabeceira do leito ou usar-se o apoio de travesseiros para sustentação } \\
\text { da cabeça do paciente. A Inspeção de cada lado deve ser feita com a cabeça ligeiramente } \\
\text { voltada para o lado oposto. À Inspeção, se as veias jugulares permanecerem túrgidas quando } \\
\text { o paciente adota a posição sentada ou semi-sentada (formando um ângulo de } 45^{\circ} \text { entre as } \\
\text { costas e o leito), está caracterizado o que se denomina ingurgitamento jugular. }\end{array}$ \\
\hline
\end{tabular}




\begin{tabular}{|c|c|}
\hline $\begin{array}{c}\text { CARACTERÍSTICAS } \\
\text { DEFINIDORAS }\end{array}$ & DEFINIÇÃO OPERACIONAL \\
\hline Mudança no padrão respiratório & $\begin{array}{l}\text { A freqüência da respiração deve ser contada a cada elevação do tórax do paciente, durante um } \\
\text { período de, no mínimo, } 15 \text { segundos, sem que ele perceba o procedimento, evitando, assim, } \\
\text { controle voluntário do movimento. A amplitude da respiração deve ser avaliada comparando-se } \\
\text { a relação da inspiração a da expiração (1:2) e associando-se ao número de movimentos } \\
\text { respiratórios por minuto (freqüência). A aferição desses parâmetros respiratórios possibilita à } \\
\text { enfermeira avaliar se houve mudança no padrão respiratório. }\end{array}$ \\
\hline Dispnéia ou respiração curta & $\begin{array}{l}\text { A entrevista e a inspeção são utilizadas na avaliação clínica da dispnéia ou respiração curta, } \\
\text { pois ela manifesta-se de duas formas: uma subjetiva, quando essa dificuldade é sentida pelo } \\
\text { paciente; e uma objetiva, evidenciada pela inspeção feita pela profissional sobre o } \\
\text { aprofundamento ou alteração da expansibilidade pulmonar (superficial ou profunda) e aumento } \\
\text { da freqüência respiratória e/ou utilização ativa da musculatura acessória da respiração } \\
\text { (músculos do pescoço na inspiração e músculos abdominais na expiração). Essa dificuldade é } \\
\text { referida pelo paciente freqüentemente associada com desconforto ou esforço exagerado, e é } \\
\text { manifestada pela sensação referida de "falta de ar" e pela constatação de respiração } \\
\text { trabalhosa. }\end{array}$ \\
\hline Ortopnéia & $\begin{array}{l}\text { A inspeção e o questionamento da posição adotada pelo paciente para respirar melhor são as } \\
\text { formas de avaliar a ortopnéia. Se ele referir a adoção de uma poltrona ou o uso de travesseiros } \\
\text { muito elevados para dormir, sem que isto seja uma atitude habitual, pode ficar caracterizada a } \\
\text { ortopnéia. }\end{array}$ \\
\hline $\begin{array}{l}\text { Sons respiratórios anormais; } \\
\text { ruídosadventícios: estertores e } \\
\text { crepitações }\end{array}$ & $\begin{array}{l}\text { A ausculta de ruídos pulmonares considerados estranhos ou adicionais aos murmúrios } \\
\text { vesiculares esperados representa o modo de avaliar essa característica definidora. Os } \\
\text { estertores e as crepitações são semelhantes ao som do rompimento de pequenas gotinhas de } \\
\text { água, podendo ser simulado pelo atrito dos fios de cabelo entre os dedos, próximo ao ouvido. } \\
\text { Esses ruídos ocorrem no final da inspiração e/ou expiração e, são melhor audíveis nas bases } \\
\text { pulmonares. }\end{array}$ \\
\hline Congestão pulmonar & $\begin{array}{l}\text { Dispnéia, ortopnéia, dispnéia paroxística noturna, tosse e edema pulmonar devem ser } \\
\text { investigados. A exemplo do derrame pleural, a palpação bimanual do tórax, sobretudo o } \\
\text { posterior, serve para detectar a dificuldade de expansão pulmonar, quando comparado um lado } \\
\text { ao outro, e a ausência do frêmito tátil, ou seja, a ausência de vibração na parede externa do } \\
\text { tórax quando o paciente repete o "trinta e três" solicitado. Além do apoio diagnóstico do estudo } \\
\text { radiológico, a percussão e a ausculta são utilizadas para a avaliação clínica do derrame pleural. } \\
\text { O acúmulo de líquidos no espaço intersticial do leito vascular pulmonar determina um som } \\
\text { maciço à percussão e estertores úmidos, mais comumente audíveis nas bases pulmonares, à } \\
\text { ausculta. A radiografia torácica pode exibir congestão vascular pulmonar, marcas aumentadas } \\
\text { de alvéolos pulmonares, alterações nos vasos sangüíneos pulmonares, dilatação cardíaca, } \\
\text { franca congestão pulmonar e derrames pleurais resultantes do aumento na pressão venosa } \\
\text { pulmonar. }\end{array}$ \\
\hline Derrame pleural & $\begin{array}{l}\text { A inspeção da traquéia deve ser feita, pois esta fica desviada da posição mediana para o lado } \\
\text { oposto ao derrame ou, quando bilateral, para o lado menos comprometido. Dispnéia, } \\
\text { taquipnéia, dor torácica e a hipoxemia (se houver comprometimento ventilatório significativo), } \\
\text { podem ser investigadas. A palpação bimanual do tórax, sobretudo o posterior, serve para } \\
\text { detectar a dificuldade de expansão pulmonar, quando comparado um lado ao outro, e a ausência } \\
\text { do frêmito tátil, ou seja, a ausência de vibração na parede externa do tórax quando o paciente } \\
\text { repete o "trinta e três" solicitado. Além do apoio diagnóstico do estudo radiológico, a percussão } \\
\text { e a ausculta, são utilizadas para a avaliação clínica do derrame pleural. O acúmulo de líquidos } \\
\text { no espaço pleural determina um som maciço à percussão e diminuição ou ausência de murmúrio } \\
\text { vesicular à ausculta. }\end{array}$ \\
\hline $\begin{array}{l}\text { Hemoglobina e hematócrito } \\
\text { diminuídos }\end{array}$ & $\begin{array}{l}\text { A aferição dos dados laboratoriais referentes à hemoglobina - } \mathrm{Hb} \text { - e ao hematócrito - } \mathrm{Ht} \text { - é } \\
\text { feita utilizando-se o raciocínio cognitivo apoiado no valor encontrado e nos respectivos padrões } \\
\text { de normalidade - Hb: } 13,5 \text { a } 18 \mathrm{~g} / \mathrm{dl} \text { no homem e } 11,5 \text { a } 16,4 \mathrm{~g} / \mathrm{dl} \text { na mulher; Ht: média de } 47 \% \\
\text { no homem e } 42 \% \text { na mulher. Ao se comparar esses valores, utiliza-se o raciocínio clínico indutivo, } \\
\text { ou seja, a partir dos padrões estabelecidos para a média da população, avalia-se o valor } \\
\text { encontrado naquele indivíduo. Quando existir um desvio desses valores para menos, diz-se que } \\
\text { a hemoglobina e o hematócrito estão diminuídos. }\end{array}$ \\
\hline Eletrólitos alterados & $\begin{array}{l}\text { A aferição das concentrações séricas de sódio, potássio, cálcio e cloro dosados em laboratório } \\
\text { é o meio de avaliação dos eletrólitos. Se os valores encontrados estiverem acima dos valores } \\
\text { considerados normais: Potássio sérico }(\mathrm{K}+): 3,5-5,0 \mathrm{mEq} / \mathrm{L} \text {; Sódio sérico (Na+): } 135-145 \\
\mathrm{mEq} / \mathrm{L} \text {; Cloro }(\mathrm{Cl}-) \text { : } 36-106 \mathrm{mEq} / \mathrm{L} \text {; Cálcio sérico }(\mathrm{Ca}++): 8,5 \text { a } 10,5 \mathrm{mg} / \mathrm{dl} \text { está caracterizada a } \\
\text { alteração de eletrólitos. }\end{array}$ \\
\hline
\end{tabular}


continuação...

\begin{tabular}{|c|c|}
\hline $\begin{array}{l}\text { CARACTERÍSTICAS } \\
\text { DEFINIDORAS }\end{array}$ & DEFINIÇÃO OPERACIONAL \\
\hline Alterações na densidade urinária & $\begin{array}{l}\text { A aferição da densidade urinária encontrada na urina, através de dosagem laboratorial, com o } \\
\text { padrão habitualmente esperado (a densidade da urina misturada de } 24 \text { horas costuma variar } \\
\text { entre } 1,016 \text { e 1,022) é o modo de avaliar se há alteração na densidade urinária. No excesso } \\
\text { de volume de líquidos é esperado um valor superior a } 1,022 \text { de densidade urinária. }\end{array}$ \\
\hline $\begin{array}{l}\text { Som cardíaco B3 (terceira bulha } \\
\text { cardíaca) }\end{array}$ & $\begin{array}{l}\text { A ausculta das bulhas cardíacas é o meio de detecção do som cardíaco B3, ou terceira bulha } \\
\text { cardíaca. Como ele é melhor audível na área mitral - 50 espaço intercostal esquerdo, na linha } \\
\text { hemiclavicular e corresponde ao ictus cordis ou "ponta do coração" - , coloca-se o paciente em } \\
\text { decúbito lateral esquerdo, e, utilizando-se a campânula (face côncava do estetoscópio), porque } \\
\text { esta bulha é um ruído de baixa freqüência, procede-se à ausculta. O som a ser ouvido pode ser } \\
\text { imitado pronunciando-se de modo rápido, soprando entre os dentes, a expressão "TU". }\end{array}$ \\
\hline Reflexo hepatojugular & $\begin{array}{l}\text { Ao fazer-se pressão sobre o fígado, de baixo para cima, as jugulares tornam-se túrgidas e } \\
\text { volumosas, voltando ao estado anterior tão logo cesse a compressão. Esta manobra é positiva } \\
\text { toda vez que houver grande estase hepática. }\end{array}$ \\
\hline Oligúria & $\begin{array}{l}\text { A aferição do balanço de líquidos representa o mais valioso critério de avaliação da oligúria e, } \\
\text { a exemplo da característica definidora ingesta maior do que o débito, a avaliação clínica faz-se } \\
\text { pelo registro de líquidos administrados/ingeridos e eliminados, utilizando-se instrumentos como } \\
\text { cálices/copos graduados ou mensurando-se periodicamente o conteúdo da bolsa coletora em } \\
\text { pacientes com cateterização vesical. O volume urinário inferior a } 20 \text { mililitros por hora (inferior } \\
\text { a } 500 \text { mililitros (ml), em } 24 \text { horas), no adulto, caracteriza a oligúria e é indicativo de insuficiência } \\
\text { renal. }\end{array}$ \\
\hline Azotemia & $\begin{array}{l}\text { A aferição das concentrações de uréia e creatinina dosadas em laboratório constitui o meio de } \\
\text { avaliação da azotemia. A azotemia está caracterizada quando os valores encontrados estiverem } \\
\text { acima dos valores considerados normais, na urina: uréia, } 20 \text { a } 40 \mathrm{~g} / 24 \text { horas e creatinina, } 1 \text { a } \\
1,5 \mathrm{~g} / 24 \text { horas; e no sangue: uréia, } 15 \text { a } 40 \mathrm{mg} \% \text { e creatinina, } 1,0 \mathrm{a} 2,0 \mathrm{mg} \% \text {. }\end{array}$ \\
\hline Mudança no estado mental & $\begin{array}{l}\text { A inspeção do estado mental é feita pelas informações dadas pelo paciente e/ou familiar sobre } \\
\text { alterações significativas do estado mental; pela observação de alterações de comportamento, } \\
\text { como hiperatividade; e, pelo questionamento sobre o que ele está sentindo, sobretudo diante } \\
\text { de situações consideradas críticas. Uma reação duradoura, diferente daquelas habitualmente } \\
\text { apresentadas pelo paciente, pode caracterizar a mudança no padrão mental, decorrente do } \\
\text { excesso de volume de líquidos. }\end{array}$ \\
\hline Agitação & $\begin{array}{l}\text { A agitação por si só não constitui uma característica definidora maior, pois ela depende da } \\
\text { investigação conjunta de outras características. Além da avaliação do estado atual do paciente, } \\
\text { deve-se obter o máximo de informação possível sobre o seu comportamento recente. Alterações } \\
\text { de comportamento como irritabilidade, mau humor, instabilidade psicomotora, inquietação, } \\
\text { precisam ser observadas, pois podem caracterizar agitação decorrente da alteração dos } \\
\text { eletrólitos, sobretudo do potássio, no excesso de líquidos. A observação do paciente e o } \\
\text { questionamento sobre hiperatividade uma característica dessa manifestação são importantes } \\
\text { componentes de ajuda e, devem ser validadas com o paciente. A comunicação estabelecida } \\
\text { entre paciente e profissional é fundamental na avaliação dessa característica definidora. }\end{array}$ \\
\hline Ansiedade & $\begin{array}{l}\text { O reconhecimento da ansiedade faz-se pela observação das manifestações psíquicas e } \\
\text { somáticas que a acompanham, tais como: inquietação, voz embargada, mãos frias e } \\
\text { sudorentas, taquicardia e boca seca. Alguns pacientes esfregam as mãos sem interrupção, } \\
\text { enquanto outros as têm trêmulas. Bocejos repetidos ou fumar incontrolavelmente podem ajudar } \\
\text { na avaliação clínica da ansiedade. A observação, a percepção (caráter interpretativo) de } \\
\text { alterações, o relacionamento com o paciente e a validação desses achados são os meios } \\
\text { clínicos de se avaliar a ansiedade. Como as demais características definidoras relacionadas a } \\
\text { reações emocionais - mudança no estado mental e agitação, a detecção da ansiedade é } \\
\text { resultado de avaliação subjetiva e, portanto, varia de pessoa para pessoa. Existe dificuldade } \\
\text { de mensura-las, o que exige, na medida do possível, uma validação dos achados pelo paciente. }\end{array}$ \\
\hline
\end{tabular}




\section{CONSIDERAÇÕES FINAIS}

As definições operacionais, das características definidoras dos diagnósticos de enfermagem são um requisito importante para estudos e desenvolvimento na prática clínica do diagnóstico Volume de Líquidos Excessivo, por favorecer o entendimento da nomenclatura às profissionais e proporcionar um impulso no direcionamento em trabalhos de pesquisa. Dessa forma, foi apresentada esta proposta de definições operacionais, referente às características definidoras do diagnóstico de enfermagem Volume de Líquidos Excessivo.

A criação de um banco de dados para mensurar adequadamente as características definidoras, com validação dessas para cada diagnóstico, poderá ajudar a formular os diagnósticos e motivar as profissionais a produzirem estudos com essa finalidade ${ }^{(18)}$.

\section{REFERÊNCIAS}

1. North American Nursing Diagnosis Association (NANDA). Diagnósticos de enfermagem da NANDA: definições e classificação 2001/2002. Porto Alegre: Artmed; 2002.

2. Grant JS, Kinney MR. The need for operational definitions for defining characteristics. Nurs Diagn 1991; 2(4):181-5.

3. Burns N, Grove S. Understanding nursing research. Philadelphia: W. B. Saunders; 1995. Understanding study variables.

4. Bevilacqua F. Fisiopatologia clínica. $3^{\mathrm{a}}$ ed. São Paulo: Atheneu; 1985.

5. Guyton AC, editor. Tratado de fisiologia médica. $7^{\mathrm{a}}$ ed. Rio de Janeiro: Guanabara Koogan; 1989.

6. McGovern M, Kuhn JK. Cardiac assessment of the elderly client. J Gerontol Nurs 1992; 18(8):40-4.

7. Smeltzer SC, Bare BG. Brunner \& Studdart: Tratado de enfermagem médico-cirúrgica. $8^{\text {a }}$ ed. Rio de Janeiro: Guanabara Koogan; 1998. Assistência a pacientes com distúrbios cardiácos.
8. Porto CC. Exame clínico: bases para a prática médica. $3^{\mathrm{a}}$ ed. Rio de Janeiro: Guanabara Koogan; 1996.

9. Miller O. Laboratório para o clínico. $8^{\mathrm{a}}$ ed. São Paulo: Atheneu; 1997.

10. Hudak CM, Gallo BM. Cuidados intensivos de enfermagem: uma abordagem holística. $6^{\mathrm{a}}$ ed. Rio de Janeiro: Guanabara Koogan; 1997.

11. Fluids \& electrolytes: made incredibly easy! Pennsylvania: Springhouse; 1997.

12. Bates B. Propedêutica médica. $6^{\mathrm{a}}$ ed. Rio de Janeiro: Guanabara Koogan; 1998.

13. Barros ALBL. O exame físico de enfermagem. In: Anais eletrônico do $6^{\circ}$ Encontro de Enfermagem e Tecnologia - ENFTEC; 1998; São Paulo. São Paulo: Centro de Estudos de Enfermagem 8 de Agosto do Hospital 9 de Julho; 1998.

14. Braunwald E, Zipes DP, Libby P. Heart disease: a textbook of cardiovascular medicine. $6^{\text {th }}$ ed. Philadelphia: W. B. Saunders; 2001.

15. Barros ALBL, Michel JLM, Lopes RS, Guimarães HCQCP. Exame do tórax - aparelho cardiocirculatório. In: Barros ALBL, organizador. Anamnese e exame físico. Porto Alegre: Artmed; 2002. p.124-33.

16. Humerez DC, Cavalcante MBG. Avaliação das condições emocionais do paciente na clínica. In: Barros ALB, organizador. Anamnese e exame físico. Porto Alegre: Artmed; 2002. p.134-54.

17. Lima AO. Métodos de laboratório aplicados à clínica: técnica e interpretação. $8^{\mathrm{a}}$ ed. Rio de Janeiro: Guanabara Koogan; 2001.

18. Guzzeta CE, editor. Clinical assessment tools for use with nursing diagnoses. St. Louis: Mosby; 1989. 